\title{
PML Expression in Soft Tissue Sarcoma: Prognostic and Predictive Value in Alkylating Agents/Antracycline-Based First Line Therapy
}

\author{
BRUNO VINCENZI,' DANIELE SANTINI,' GAIA SCHIAVON,' ANNA MARIA FREZZA,' \\ ANTONIO RUSSO, ${ }^{4 *}$ JAMES E. BUTRYNSKI, ${ }^{6}$ AND GIUSEPPE TONINI ${ }^{1}$ \\ 'Department of Medical Oncology, University Campus Bio-Medico, Roma, Italy \\ ${ }^{2}$ Istituto Nazionale dei Tumori, Milano, Italy \\ ${ }^{3}$ Department of Pathology, General Hospital, Treviso, Italy \\ ${ }^{4}$ Department of Surgical and Oncological Sciences, Università di Palermo, Palermo, Italy \\ ${ }^{5}$ Department of Human Pathology, Università di Palermo, Palermo, Italy \\ ${ }^{6}$ Soft Tissue and Bone Sarcoma Department, Dana Farber Cancer Institute, Boston, Italy
} MARIANNA SILLETTA,' PIERFILIPPO CRUCITTI,' PAOLO CASALI, ${ }^{2}$ ANGELO P. DEI TOS, ${ }^{3}$ SABRINA ROSSI, ${ }^{3}$ SERGIO RIZZO, ${ }^{4}$ GIUSEPPE BADALAMENTI, ${ }^{4}$ ROSA MARIA TOMASINO, ${ }^{5}$

\begin{abstract}
Soft tissue sarcomas are aggressive tumors representing $<1 \%$ of all adult neoplasms. Aim of our study was to evaluate promyelocytic leukemia gene expression value as prognostic factor and as a factor predicting response to alkylating agents/antracycline-based first line therapy. One hundred eleven patients affected by locally advanced and metastatic soft tissue sarcoma were selected. PML expression was evaluated by immunohistochemical analysis in pathological samples and in the corresponding normal tissue from each case. PML immunohistochemical results were correlated with prognosis and with radiological response to alkylating agents/antracycline-based first line therapy. PML expression was significantly reduced in synovial sarcomas $(P<0.000 \mathrm{I})$, in myofibroblastic sarcomas $(P<0.000 \mathrm{I})$, angiosarcomas $(P<0.000 \mathrm{I})$, in leiomyosarcomas $(P=0.003)$, in mixoid liposarcomas $(P<0.000 \mathrm{I})$, and in dedifferentiated liposarcomas $(P<0.000 \mathrm{I})$. No significant difference was found for pleomorphic sarcoma [3I.8 (95\% Cl: $16.7-41.0) ; P=0.2 \mathrm{I}]$. and pleomorphic liposarcomas $(P=0.5 \mathrm{I})$. Loss of PML expression was found to be statistically correlated with $\operatorname{TTP}(P<0.000 \mathrm{I})$, median duration of response $(P=0.007)$, and $O S(P=0.02)$. No correlation was observed between PML expression and treatment efficacy. PML IHC expression is down-regulated in synovial sarcomas, myofibroblastic sarcomas, angiosarcomas, liposarcoma, and leiomyosarcomas and its expression correlated with prognosis.
\end{abstract}

J. Cell. Physiol. 227: I657-I662, 20I2. (C) 20II Wiley Periodicals, Inc.

PML is known to be involved in the pathogenesis of acute promyelocytic leukaemia, which is characterized by the $t(15 ; 17)$ traslocation that brings to the juxtaposition between PML gene, located in 15q22 locus, and the gene encoding for the retinoid acid receptor $\alpha$, on chromosome 17. The two fusion proteins deriving from the $t(15 ; \mid 7)$ traslocation (PML-RAR $\alpha$ and RAR $\alpha$ $\mathrm{PML}$ ) lead to a differentiation blockage and a shorter survival of myeloid precursors (Melnick and Licht, 1999).

PML gene consists of nine exons from which, through alternative splicing, seven different isoforms originate, both nuclear (PML I, II, III, IV, V, VI) and citoplasmatic (VII) (Salomoni and Bellodi, 2007). PML nuclear isoforms, marked by the presence of nuclear localization signal encoded in exon 6 , can both localize in nucleoplasm or into complex multiproteinaceus structures called PML nuclear bodies (PML-NBs). PML-NBs recognize both constitutive elements, represented by high ordered PML multimers and SI00 monomers, and components which provisionally place in PML-NBs and interact with PML itself such as p53, DAXX, c-Jun, pRB, mTOR (WeidtkampPeters et al., 2008). PML-NBs formation and the interactions between PML and its different protein partners become possible thanks to post-traslational modifications among which small ubiquitin-like modifiers (SUMO) emerged to be particularly relevant (Shen et al., 2006).

Although today some of the biological aspects of PML action into PML-NBs are still not clear, different studies underline the importance of PML in the regulation of apoptosis, cellular proliferation and senescence, pointing out a possible involvement of this oncosuppressor in the pathogenesis of different non-hematological neplasms.

Soft tissue sarcomas are defined as a group of rare tumors originating from non-epithelial extraskeletal tissues, including muscle, fat and fibrous structures, deriving mainly from

*Correspondence to: Antonio Russo, Department of Surgical and Oncological Sciences, Section of Medical Oncology; Università di Palermo, Via del Vespro 127 - 90127 Palermo, Italy.

E-mail: lab-oncobiologia@usa.net

Received 16 April 201 I; Accepted 3 June 2011

Published online in Wiley Online Library (wileyonlinelibrary.com), I5 June 201 I.

DOI: 10.1002/jcp.22889 
embryonic mesoderm with a partial neuroectodermal contribution. These tumors can arise anywhere in the body (although limb, limb girdle, and abdomen are the most frequent sites) and appear to be extremely heterogeneous, including more than 50 different histotypes (Clark et al., 2005). Regarding epidemiologic aspects, soft tissue sarcomas account for $<1 \%$ of all human tumors in the adult, with an annual incidence of 10,390 new cases in United States. The median age at the diagnosis is 57 years and the onset of these tumors in the III-IV decade is not rare (Ries et al., 2007).

The treatment of soft tissue sarcomas consists of a multimodal approach deriving from the integration of surgery, radiotherapy, and standard chemotherapy. The management of locally advanced and metastatic soft tissue sarcomas is today founded on standard chemotherapy. Ifosfamide plus doxorubicin-based regimens are routinely used in this setting as first line treatment. Unfortunately, despite the advances done in the last 10 years with the introduction of new target drugs, up to date soft tissue sarcomas become metastatic into 2 years in $80 \%$ of patients, with a 5-years overall survival (OS) of 25-30\% (Ries et al., 2007). Given these data, the identification of prognostic elements and reliable factors predicting the efficacy of alkylating agents/antracycline-based therapy appears necessary, in order to better identify those patients who could benefit from a standard chemotherapy and those who require alternative approaches.

Aim of this retrospective study was to assess PML immunohistochemical expression in six different subtypes of soft tissue sarcoma (synovial sarcoma, myofibroblastic sarcoma, angiosarcoma, liposarcoma, leiomyosarcoma, pleomorphic sarcoma) and evaluate PML down-regulation as a predictive factor predicting of resistance to alkylating agents/ antracycline-based first line therapy in patients affected by locally advanced and metastatic soft tissue sarcomas.

\section{Materials and Methods}

Tumor specimen acquisition and histological examination

Samples sources. One hundred eleven cases diagnosed between September 2007 and November 2008 have been collected from the records of Pathology Departments of Regional Hospital of Treviso, Campus Bio-Medico of Rome and University of Palermo. Only cases with available paraffin blocks were selected. All the clinical data were reviewed and a reclassification of all the cases according to the new criteria for diagnosis (WHO) was performed.

Assembly of TMA. A hematoxylin- and eosin-stained (H\&E) section from each primary tumor was prepared and areas of representative non-necrotic neoplasm circled on coverslip. Using a Beecher Instrument (Silver Spring, MD), TMAs were constructed introducing three cores of I mm diameter of selected tumor region and two cores of corresponding normal tissue from each case. Following tissue microarray (TMA) construction, H\&E stained section of the TMA recipient block was prepared and reviewed to confirm the presence of intact neoplasm. Sections of $5 \mu \mathrm{m}$ were prepared in order to perform $\mathrm{H} \& \mathrm{E}$ stain as well as IHC staining.

Immunohistochemical analysis. IHC was performed by the streptavidin-biotin method. Endogenous peroxidase in the sections was blocked by incubating them in $3 \%$ hydrogen peroxide. A rabbit polyclonal antibody against PML protein (clone PG-M3; Santa Cruz Biotecnology, Inc, Santa Cruz, CA) was used at I:50 dilution. This antibody has been used and validated previously by our group and by others (Lee et al., 2007; Vincenzi et al., 2009). Sections were incubated with LSAB2 (Dakocytomation). 3-3'-diaminobenzidine (DAB) was used for color development and hematoxylin was used for counterstaining. Negative control TMA slides processed without primary antibody were prepared. Slides were examined without knowledge of the corresponding clinico-pathologic data.

Immunostaining was considered positive if appropriate brown staining was seen in cellular nucleus. PML expression was established calculating the percentage of nuclear immunoreactive cells out of the total cells counted. The immunostaining evaluation was conducted by two different pathologist, both of which were not aware of patients clinical data. In terms of PML IHC results, all cases were classified as follows: complete loss (nuclear immunoreactivity in $<10 \%$ of tumor cells), focal positivity (in $\geq 10 \%$ but $<50 \%$ ), and diffuse positivity (in $\geq 50 \%$ ), as previously reported by Lee et al. (2007).

\section{Statistical analysis}

Descriptive analysis was performed using median values and $95 \%$ confidence interval $(\mathrm{Cl})$. Differences between tumor tissue and normal surrounding tissue were assessed by using the Wilcoxon's test for non-parametric-dependent continuous variables.

SPSS software (version 17.00; SPSS, Chicago, IL) was used for statistical analysis. A $P$-value of $<0.05$ was considered to indicate statistical significance.

The time-to-progression (TTP) analysis was calculated as the period from the date of starting the treatment to the first observation of disease progression or to death from any cause within 60 days after the start of treatment or the most recent tumor assessment. The OS time was calculated as the period from the date of starting treatment until death from any cause or until the date of the last follow-up, at which point data were censored. TTP and OS were both determined by Kaplan-Meier product-limit method (Kaplan and Meier, 1958). Stratified permutation tests were carried out to explore the association between tumor response and PML down-regulation. The differences in terms of TTP and OS according to the presence of PML down-regulation were evaluated by the log-rank test (Peto et al., 1977). The Cox proportional hazards model was applied to the multivariate survival analysis (Cox, 1972).

Results

PML immunohistochemical expression in soft tissue sarcomas

PML IHC expression was evaluated in six different subtypes of soft tissue sarcoma (synovial sarcoma, myofibroblastic sarcoma, angiosarcoma, liposarcoma, leiomyosarcoma, pleomorphic sarcoma) and in the corresponding normal tissue samples (Table I). PML IHC expression resulted focally or completely lost in most of the pathological samples (Fig. I), with the exception of pleomorphic sarcoma and pleomorphic liposarcoma: particularly, no angiosarcoma, synovial sarcoma, or mixoid liposarcoma samples showed a diffuse PML positivity (Table 2).

PML expression was significantly reduced in synovial sarcoma specimens, with a median expression percentage of I0.3\% (95\% Cl: $3.98-2 \mathrm{I} .06)$ and a value of $40.8 \%$ (95\% Cl: 26.87-54.5; $P<0.000 \mathrm{I}$ ) in the corresponding normal tissue. Furthermore, a significantly lower PML median expression was found in myofibroblastic sarcoma [18.5\% (95\% Cl: 12.5-26.7) vs. $41.7 \%$ (95\% Cl: $24.9-58.7) ; P<0.000 \mathrm{I}]$ and in angiosarcoma [I5.8\% (95\% Cl: $4.7-20.6)$ vs. $39.0 \%$ (95\% Cl: $24.8-58.30$;

TABLE I. Patients features

\begin{tabular}{lc}
\hline Median age & 59 years \\
Range & $24-81$ years \\
Sex, male/female & $61 / 50$ \\
ECOG performance status & \\
0 & 62 \\
I & 42 \\
2 & 7 \\
Prior adjuvant chemotherapy & 24 \\
Prior adjuvant chemotherapy & 35 \\
Histology & \\
Liposarcoma & 34 \\
Leiomyosarcoma & 15 \\
Angiosarcoma & 9 \\
Synovial sarcoma & 25 \\
Miofibroblastic sarcoma & 13 \\
Pleomorphic sarcoma & 15 \\
\hline
\end{tabular}




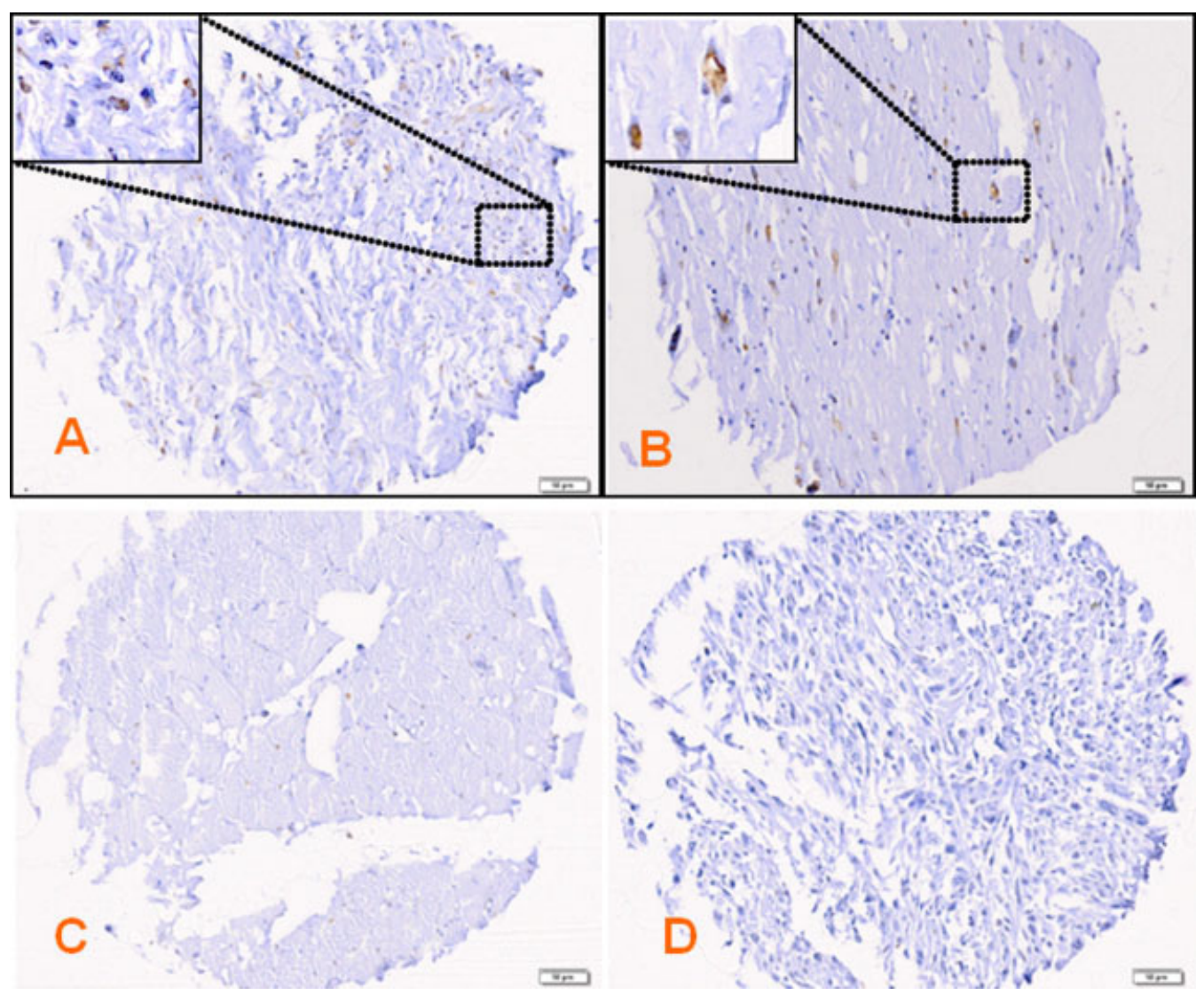

Fig. I. PML immunohistochemical expression in normal tissue ( $A$ and $C$ ) and its down-regulation in soft tissue sarcomas (B and $D)$. [Color figure can be seen in the online version of this article, available at http://wileyonlinelibrary.com/journal/jcp]

$P<0.000$ I] when compared with the normal tissue samples. A statistically significant difference was also observed between PML median expression in leiomyosarcoma and normal tissue control (2I\% [95\% Cl: I0.5-3I.2] vs. 40.5\% [95\% Cl: 26.9-6I.8]; $P=0.003)$.

On the contrary, no significant difference in median PML expression was found between pleomorphic sarcoma specimen [3I.8 (95\% Cl: |6.7-4|.0)] and normal tissue control [38.7 (95\% Cl: 24.9-55.3); $P=0.21$ ]

As for liposarcoma, PML median expression appeared significantly reduced in mixoid liposarcoma [ $1 \mathrm{I} .4 \%(95 \% \mathrm{Cl}$ : 6.9-18.5) vs. $42.7 \%$ (95\% Cl: $26.0-52.0) ; P<0.000 I]$ and in dedifferentiated liposarcoma [I7.6\% (95\% Cl: II.5-29.8) vs. $39.8 \%$ (95\% Cl: 24.6-58.2); $P<0.0001$ ], while no significant difference was detected between pleomorphic liposarcoma and normal tissue specimens [36.0 (95\% Cl: I8.8-46.I) vs. 38.6 (95\% Cl: 23.4-53.8); $P=0.5 \mathrm{I}]$.

\section{Correlation between PML expression and treatment efficacy}

Patients with complete loss of PML expression showed a median TTP of 3.50 months (95\% Cl: 1.78-4.93), compared with 7.00 months $(95 \% \mathrm{Cl}: 3.5 \mathrm{I}-\mathrm{I} 2 . \mathrm{II})$ in those with a focally and diffuse $P M L$ positivity $(P<0.000 I)$. A statistically significant difference was observed also in terms of median duration of response, which resulted 1.8 months $(95 \% \mathrm{Cl}$ : $1.01-5.80)$ in patients with complete loss of PML expression and 7.5 months (95\% Cl: 4.33-10.34) in those with partial PML loss. $(P=0.007)$. Finally, overall survial (OS) was found to be significantly lower in patients with complete loss of PML when compared with those having a focally and diffuse positivity [ 9.8 months ( $95 \% \mathrm{Cl}: 5.67-$ $18.80)$ vs. 17.50 months ( $95 \% \mathrm{Cl}$ : I I .50-23.30); $P=0.02]$. All these data are summarized in Table 3 and Kaplan-Meier curves are shown in Figure 2.

TABLE 2. Immunohistochemical staining for PML protein in soft tissue sarcoma

\begin{tabular}{lccr}
\hline & PML complete loss (\%) & PML focal positivity (\%) & PML diffuse positivity (\%) \\
\hline Synovial sarcoma (25 samples) & $16(64)$ & $9(36)$ & 0 \\
Myofibroblastic sarcoma (13 samples) & $5(38.5)$ & $6(46.1)$ & $2(15.4)$ \\
Liposarcoma (34 samples) & $14(41.2)$ & $15(44.1)$ & $5(14.7)$ \\
Myxoid (12 samples) & $7(58.3$ & $5(41.7)$ & 0 \\
Dedifferentiated (8 samples) & $5(62.5)$ & $2(25)$ & $1(12.5)$ \\
Pleomorphic (14 samples) & $3(21.4)$ & $6(42.9)$ & $5(35.7)$ \\
Pleomorphic sarcoma (15 samples) & $4(26.7)$ & $7(40)$ & $5(33.3)$ \\
Leiomyosarcoma (15 samples) & $6(40)$ & $4(46.7)$ & $2(13.3)$ \\
Angiosarcoma (9 samples) & $5(55.6)$ & $4(44.4)$ & 0 \\
\hline
\end{tabular}


TABLE 3. PML down-regulation influence on TTP, duration of response and $O S$ in locally advanced and metastatic soft tissue sarcoma

\begin{tabular}{|c|c|c|}
\hline TTP & $\begin{array}{c}\text { Median TTP } \\
(95 \% \mathrm{Cl}) \text { in months }\end{array}$ & $P$-value \\
\hline $\begin{array}{l}\text { PML expression complete loss } \\
\text { PML expression partial loss }\end{array}$ & $\begin{array}{l}3.50 \text { (95\% Cl: I.78-4.93) } \\
7.00 \text { (95\% Cl: } 3.5 \mathrm{I}-12.1 \mathrm{I})\end{array}$ & $<0.0001$ \\
\hline Duration of response & $\begin{array}{l}\text { Median duration of response } \\
(95 \% \mathrm{Cl}) \text { in months }\end{array}$ & \\
\hline $\begin{array}{l}\text { PML expression complete loss } \\
\text { PML expression partial loss }\end{array}$ & $\begin{array}{l}\text { I.8 (95\% Cl: } 1.0 \mathrm{I}-5.80) \\
7.5 \text { (95\% Cl: } 4.33-10.34)\end{array}$ & 0.007 \\
\hline os & Median OS $(95 \% \mathrm{Cl})$ in months & \\
\hline $\begin{array}{l}\text { PML expression complete loss } \\
\text { PML expression partial loss }\end{array}$ & $\begin{array}{l}9.80 \text { (95\% Cl: } 5.67-18.80) \\
17.50 \text { (95\% Cl: | | I.50-23.30) }\end{array}$ & 0.02 \\
\hline
\end{tabular}

Regarding the correlation between PML immunohistochemical expression and radiologic response to therapy, a CT scan after the third cycle of treatment in patients with complete PML loss showed complete response in $4.6 \%$ of all cases, partial response in $32.6 \%$, stable disease in $37.2 \%$, and progression of disease in $25.6 \%$. In patients with a partial loss of PML expression we observed a partial response in $23.5 \%$ of cases, stable disease in $45.6 \%$, and progression of disease in $30.9 \%$, with no complete responses among these patients. All these data have been reported in Table 4 .

\section{Discussion}

Although PML oncosuppressive role was hypothesized since its identification at the breakpoint of $\mathrm{t}(15 ; 17)$ translocation, growing evidences in literature are today confirming it. Given its multiple interactions in the context of PML-NBs, allowed by PML post-traslational modifications especially lysine SUMOylation in three different sites, PML seems to play a crucial role in the control of apoptosis, cellular proliferation and senescence, and protein synthesis.

PML involvement in apoptosis regulation emerged with a first study by Wang et al. (1998) which analyzed the survival of PML wild type and PML knocked-down mice and the viability of splenocytes after $\gamma$-irradiation. PML-/- mice were protected from $\gamma$-irradiation-induced death compared with $\mathrm{Pml}+\mathrm{I}+$ mice $(P<0.02)$ and apoptosis in their splenocytes was significantly lower, confirming PML requirement in DNA-damage-induced apoptosis. Although the mechanisms through which PML regulates apoptosis are not completely clear yet, the interactions with $\mathrm{p} 53$, DAXX, and c-Jun seem to be particularly relevant. PML has been proven to regulate $\mathrm{p} 53$ stability, by interfering with Mdm2 ubiquitination (Kurki et al., 2003), activity by regulating acetylation (Halazonetis et al., 2008), and its capability to induce the transcription of apoptosis intermediaries by increasing HIPK2-mediated p53 phosphorylation (Moller et al., 2003). The interaction between PML and DAXX causes a cellular sensitization to Fas-induced apoptosis (Torii et al., 1999) and blocks the nuclear transcriptional repression usually induced by DAXX ( $\mathrm{Li}$ et al., 2000). Finally, PML has been found to modulate c-Jun proapoptotic function by activating JNK-dependent phosphorylation (Salomoni et al., 2005).

Different studies have recently underlined how PML overexpression leads to a cell cycle arrest, mainly in GI phase (Le et al., I996; Mu et al., 1997). Cellular senescence, meant as a permanent exclusion by cellular cycle, is regulated by PML through its interactions with p53 (as previously reported) and PRB (Wang et al., 1998), whose levels are increased by PML overexpression (Ferbeyre et al., 2000; Gurrieri et al., 2004) and whose repressive function results strengthened (through a HDAC-mediated mechanism; Mallette et al., 2004).

Interestingly, PML seems to be also involved in the control of protein synthesis, by causing a nuclear retention of the eukaryotic translation initiation factor elF4E (Cohen et al., 200 I; Culjkovic et al., 2007), and angiogenesis, by modulating hypoxia inducible factor $\alpha$ levels through the mTOR pathway (Bernardi et al., 2006).

Despite these emerging evidences, few studies analyzed PML role in human tumors. Gurrieri et al. evaluated PML IHC expression in different histological subtypes of human neoplasia and detected a reduced or abolished PML expression in prostate adenocarcinomas (63\% and $28 \%$, respectively), colon adenocarcinomas (31\% and $17 \%$ ), breast carcinomas $(21 \%$ and $31 \%$ ), lung carcinomas (36\% and $21 \%$ ), lymphomas (14\% and $69 \%)$, CNS tumors (24\% and $49 \%$ ), and germ cell tumors ( $36 \%$ and $48 \%$ ), but not in thyroid or adrenal carcinomas.
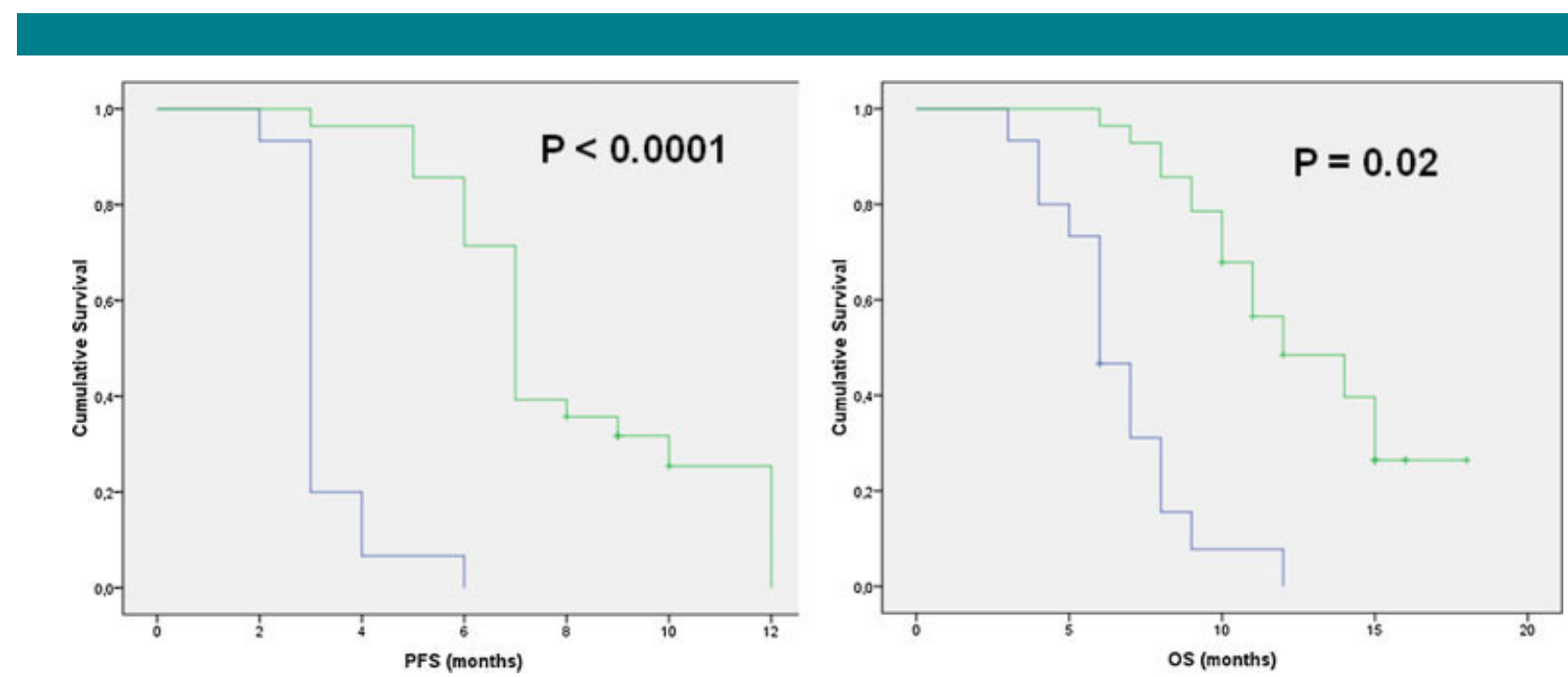

Fig. 2. Kaplan-Meier survival curves for soft tissue sarcoma patients, according to PML immunohistochemical expression. PML down-regulation in soft tissue sarcoma specimens was associated with a statistically significantly lower progression-free survival $(P<0.000 I)$ and overall survival $(P=0.02)$. [Color figure can be seen in the online version of this article, available at http://wileyonlinelibrary.com/journal/jcp] 
TABLE 4. PML immunohistochemical expression and radiologic response to epirubicin/ifosfamide first line therapy $(n=145, I I I)$

\begin{tabular}{lccrr}
\hline & CR (\%) & PR (\%) & SD (\%) & PD (\%) \\
\hline PML expression complete loss (50 patients) & $2(4.6)$ & $16(32.6)$ & $19(37.2)$ & $13(25.6)$ \\
PML diffuse expression or partial loss (6I patients) & 0 & $14(23.5)$ & $28(45.6)$ & $19(30.9)$ \\
\hline
\end{tabular}

$\mathrm{CR}$, complete response; $\mathrm{PR}$, partial response; SD, stable disease; $\mathrm{PD}$, progression of disease.

Furthermore, the authors underlined how PML loss was also associated with tumor progression in prostate adenocarcinomas (progression from prostatic intraepithelial neoplasia to invasive carcinoma was associated with complete PML loss; $P<0.001$ ), breast cancer (complete PML loss was associated with lymph node metastasis; $P=0.01$ ), and CNS tumors (complete PML loss was associated with high-grade tumors; $P=0.003$; Gurrieri et al., 2004). PML IHC expression has been found to be reduced or abolished also in a minority of gastric carcinomas (31.7\% and $10.6 \%$, respectively) and its complete loss resulted associated with more lymphatic invasion, higher PTNM stage, and worse patients survival (Lee et al., 2007). Finally, a study from our group evaluated PML expression in ampullary carcinoma: in $38.7 \%$ of the specimens, PML was classified as absent, in $25.8 \%$ as focally expressed, and in $2235.5 \%$ as diffusely expressed. Furthermore, disease-free survival (DFS) and OS were significantly influenced by $P M L$ expression ( $P=0.00 \mathrm{I}$ and $P=0.002$, respectively). By a multivariate analysis, PML expression was the strongest prognostic factor for DFS $(P=0.003)$ and the only statically significant prognostic factor for $O S(P=0.009)$ (Vincenzi et al., 2009).

PML possible role in sarcomatous transformation has been postulated by Huang et al. (2008), through the study of chromosomal aberrations in malignant diffuse-type tenosynovial giant cell tumors (D-TSGCTs). In fact the deletion at I5q, the locus containing PML gene, represented the most frequent non-random chromosomal alteration in malignant $D$ TSGCTs (Huang et al., 2008). Up to date, few is known about PML expression in soft tissue sarcoma and no predictive factors are available at the moment in order to select those patients who could benefit from a standard first line chemotherapy or those who need a different approach. Single agents (doxorubicin, ifosfamide, dacarbazine) and anthracycline-based combination regimens (doxorubicin or epirubicin with ifosfamide and/or dacarbazine) have been widely used in the metastatic stage and today epirubicin $\left(30 \mathrm{mg} / \mathrm{m}^{2}\right.$ day $\left.\mathrm{I}-3\right) /$ ifosfamide $\left(3,000 \mathrm{mg} / \mathrm{m}^{2}\right.$ day l-3)/mesna $\left(3,000 \mathrm{mg} / \mathrm{m}^{2}\right.$ day l-3) represents a valid possible first line therapy in this setting, according to NCCN guidelines.

A previous smaller study from our group (Vincenzi et al., 2010) has already pointed out how PML expression was significantly down-regulated in soft tissue sarcoma. The present study assessed PML immunohistochemical expression in a wider popolation (I I I cases of synovial sarcoma, myofibroblastic sarcoma, angiosarcoma, liposarcoma, leiomyosarcoma, and pleomorphic sarcoma) and evaluated, for the first time, the possible role of PML down-regulation as a factor predicting resistance to alkylating agents/anthracyclinebased first line therapy in patients affected by locally advanced and metastatic soft tissue sarcoma. The results underline how PML down-regulation represents a common occurrence in soft tissue sarcoma different histotypes, suggesting a possible pathogenetic role of the gene itself in the tumorigenesis of these complex tumors. In concordance with previous studies, PML immunohistochemical expression was found to be partially or completely loss in some histotypes of soft tissue sarcoma (synovial sarcoma, myofibroblastic sarcoma, angiosarcoma, myxoid, and dedifferentiated liposarcoma, leiomyosarcoma) but not in other (pleomorphic sarcoma and pleomorphic liposarcoma). The most significative results have been obtained by comparing PML expression in angiosarcoma and synovial sarcoma specimens with the normal tissue samples. Of interest, the expression of PML protein was found to be different among the three subtypes of liposarcoma analysed: it resulted extremely reduced in dedifferentiated and myxoid liposarcoma but not in the plemorphic, pointing out once more how liposarcoma subtypes (pleomorphic, dedifferentiated, and myxoid) should be considered as different pathological entities.

The preliminary data concerning PML predictive value in patients treated with alkylating/anthracycline-based first line therapy proved to be interesting as well. In those patients with PML quantitative down-regulation, a worse outcome was revealed in terms of TTP and duration of response, confirming $\mathrm{PML}$ value as a factor predicting resistance to standard alkylating/anthracycline-based first line therapy. Given the statistically significant difference obtained in terms of OS between patients with a partial or complete loss of PML expression ( 9.8 months) and those whose expression was preserved (17.5 months), it is reasonable postulate a possible prognostic significance of PML in patients affected by soft tissue sarcoma, as also suggested by previous studies. On the contrary, it does not seem to be any significant correlation between PML expression and the radiologic evaluation of the treatment response throughout RECIST criteria.

Despite the relevance of the data previously reported, the present study validity is limited by its retrospective nature and the small sample size. Moreover, it is clear today that the term "soft tissue sarcomas" includes several different entities, that have been proven to be characterized by a different biological behavior, prognosis, and treatment approach: therefore, PML value as a prognostic and predictive factor should be assessed apart in the different sarcoma subtypes, starting from the most frequent ones (such as synovial sarcoma, leiomyosarcomas, and liposarcomas). Further studies are needed to confirm these results, to corroborate PML significance as a prognostic factor and to prospectically evaluate PML predictive value in the different sarcoma histotypes.

\section{Literature Cited}

Bernardi R, Guernah I, Jin D, Grisendi S, Alimonti A, Teruya-Feldstein J, Cordon-Cardo C Simon MC, Rafii S, Pandolfi PP. 2006. PML inhibits HIF-Ialpha translation and neoangiogenesis through repression of $\mathrm{mTOR}$. Nature 442:779-785.

Clark MA, Fisher C, Judson I, Thomas JM. 2005. Soft-tissue sarcomas in adults. N Engl J Med 353:70I-7II.

Cohen N, Sharma M, Kentsis A, Perez JM, Strudwick S, Borden KL. 200I. PML RING suppresses oncogenic transformation by reducing the affinity of elF4E for mRNA. Embo J 20:4547-4559.

Cox DR. 1972. Regression models and life tables. J R Stat Soc B 34:187-220.

Culjkovic B, Topisirovic I, Borden KL. 2007. Controlling gene expression through RNA regulons: The role of the eukaryotic translation initiation factor elF4E. Cell Cycle 6:65-69. Ferbeyre G, de Stanchina E, Querido E, Baptiste N, Prives C, Lowe SW. 2000. PML is induced by oncogenic ras and promotes premature senescence. Genes Dev 14:20I5-2027. Gurrieri C, Capodieci P, Bernardi R, Scaglioni PP, Nafa K, Rush LJ, Verbel DA, Cordon-Cardo C, Pandolfi PP. 2004. Loss of the tumor suppressor PML in human cancers of multiple histologic origins. J Natl Cancer Inst 96:269-279.

Halazonetis TD, Gorgoulis VG, BartekJ. 2008. An oncogene-induced DNA damage model for cancer development. Science 319:1352-1355.

Huang HY, West RB, Tzeng CC, van de Rijn M, Wang JW, Chou SC, Huang WW, Eng HL, Lin CN, Yu SC, Wu JM, Lu CC, Li CF. 2008. Immunohistochemical and biogenetic features of diffuse-type tenosynovial giant cell tumors: The potential roles of cyclin A, P53, and deletion of $15 q$ in sarcomatous transformation. Clin Cancer Res 14:6023-6032.

Kaplan E, Meier P. 1958. Non parametric estimation from incomplete observations. J Am Stat Assoc 53:457-481.

Kurki S, Latonen L, Laiho M. 2003. Cellular stress and DNA damage invoke temporally distinct Mdm2, p53 and PML complexes and damage-specific nuclear relocalization. J Cel Sci I 16:3917-3925. 
Le XF, Yang P, Chang KS. 1996. Analysis of the growth and transformation suppressor domains of promyelocytic leukemia gene, PML. J Biol Chem 27I:I30-135.

Lee HE, Jee CD, Kim MA, Lee HS, Lee YM, Lee BL, Kim WH. 2007. Loss of promyelocytic leukemia protein in human gastric cancers. Cancer Lett 247:103-109.

Li H, Leo C, Zhu J, Wu X, O'Neil J, Park EJ, Chen JD. 2000. Sequestration and inhibition of Daxx-mediated transcriptional repression by PML. Mol Cell Biol 20:1784-1796.

Mallette FA, Goumard S, Gaumont-Leclerc MF, Moiseeva O, Ferbeyre G. 2004. Human fibroblasts require the $\mathrm{Rb}$ family of tumor suppressors, but not $\mathrm{p} 53$, for PML-induced senescence. Oncogene 23:91-99.

Melnick A, Licht JD. 1999. Deconstructing a disease: RARalpha, its fusion partners, and their roles in the pathogenesis of acute promyelocytic leukemia. Blood 93:3167-3215.

Moller A, Sirma H, Hofmann TG, Rueffer S, Klimczak E, Droge W, Will H, Schmitz ML. 2003. $\mathrm{PML}$ is required for homeodomain-interacting protein kinase 2 (HIPK2)-mediated $\mathrm{p} 53$ phosphorylation and cell cycle arrest but is dispensable for the formation of HIPK domains. Cancer Res 63:4310-4314.

Mu ZM, Le XF, Vallian S, Glassman AB, Chang KS. 1997. Stable overexpression of PML alters regulation of cell cycle progression in HeLa cells. Carcinogenesis 18:2063-2069.

Peto R, Pike MC, Armitage P, Breslow NE, Cox DR, Howard SV, Mantel N, McPherson K. PetoJ, Smith PG. 1977. Design and analysis of randomized clinical trials requiring prolonged observation of each patient. II. analysis and examples. Br J Cancer 35:I-39.

Ries LAG, Melbert D, Krapcho M, Stinchcomb DG, Howlader N, Horner MJ, Mariotto A Miller BA, Feuer EJ, Altekruse SF, Lewis DR, Clegg L, Eisner MP, Reichman M, Edwards BK. (eds). SEER Cancer Statistics Review, 1975-2005, National Cancer Institute.
Bethesda, MD, http://seer.cancer.gov/csr/1975_2005/ based on November 2007 SEER data submission, posted to the SEER web site, 2008 .

Salomoni P, Bellodi C. 2007. New insights into the cytoplasmic function of PML. Histol Histopathol 22:937-946.

Salomoni P, Bernardi R, Bergmann S, Changou A, Tuttle S, Pandolfi PP. 2005. The promyelocytic leukemia protein PML regulates c-Jun function in response to DNA damage. Blood 105:3686-3690.

Shen TH, Lin HK, Scaglioni PP, Yung TM, Pandolfi PP. 2006. The mechanisms of PML-nuclear body formation. Mol Cell 24:331-339.

Torii S, Egan DA, Evans RA, Reed JC. 1999. Human Daxx regulates Fas-induced apoptosis from nuclear PML oncogenic domains (PODs). Embo J 18:6037-6049.

Vincenzi B, Santini D, Perrone G, Russo A, Adamo V Rizzo S, Castri F, Antinori A, Alloni R, Crucitti PF, Morini S, Rabitti C, Vecchio FM, Magistrelli P, Coppola R, Tonini G. 2009. Promyelocytic leukemia (PML) gene expression is a prognostic factor in ampullary cancer patients. Ann Oncol 20:78-83.

Vincenzi B, Perrone G, Santini D, Grosso F, Silletta M, Frezza A, Rossi S, Russo A, Rabitti C Gebbia N, Badalamenti G, Casali P, Muda AO, Dei Tos AP, Tonini G. 20I0. PML downregulation in soft tissue sarcomas. J Cell Physiol 224:644-648.

Wang ZG, Ruggero D, Ronchetti S, Zhong S, Gaboli M, Rivi R, Pandolfi PP. 1998. PML is essential for multiple apoptotic pathways. Nat Genet 20:266-272.

Weidtkamp-Peters S, Lenser T, Negorev D, Gerstner N, Hofmann TG, Schwanitz G, Hoischen C, Maul G, Dittrich P, Hemmerich P. 2008. Dynamics of component exchange at PML nuclear bodies. J Cell Sci I21:273 I-2743. 